\title{
INFLUENCE OF SULPHUR OXIDIZING FUNGI ON GROWTH AND YIELD OF SOYBEAN
}

Kuldeepsingh Thakur

College of Agriculture Nagpur (Dr. P D K V, Akola) Maharashtra, India Corresponding author Email : kdthakur60@gmail.com

\begin{abstract}
:
Efficacy of two sulphur oxidizing fungal cultures viz., Trichoderma harzianum and Aspergillus niger individually and in combination with sulphur was evaluated in soybean crop in field trial at research farm of Plant Pathology Section, College of Agriculture, Nagpur, during kharif, 2012. The yield parameters viz., number of pods plant-1, grain yield and 1000 grains weight increased significantly due to inoculation of cultures under study with or without sulphur fertilizer. The treatment of T. harzianum and recommended dose of fertilizer (30:75:30 NPK kg ha-1) with sulphur (15 kg ha-1) had given maximum response in respect of number of pods plant-1, grain yield and 1000 grains weight. The number of pods plant-1, grain yield and 1000 grains weight were increased from 47.30 to $68.34,823.00$ to $1590.00 \mathrm{~kg}$ ha- 1 and 111.21 to $127.43 \mathrm{~g}$ by this treatment respectively over uninoculated control. The inoculation of $\mathrm{T}$. harzianum + Sulphur (15 kg ha-1) + RDF could increase available sulphur content in soil from $13.36 \mathrm{~kg}$ ha-1 in control to a maximum of $19.60 \mathrm{~kg}$ ha-1 and sulphur uptake ranging from 250.00 to $322.33 \mathrm{mg} 100 \mathrm{~g}-1$ plant dry weight when compared with control.
\end{abstract}

\section{Keywords:}

Sulphur oxidizing fungi

\section{Introduction:}

Soybean [Glycine max (L.) Merrill] is well known oilseed as well as pulse crop which is grown in various countries. Pulses and oilseeds are important constituents of Indian diet and supply a major part of the protein requirement. Soybean supplies 20 per cent oil and 40 per cent protein. Sulphur is one of the seventeen essential nutrients and it is a structural component of amino acids, proteins, vitamins and enzymes and is essential to produce chlorophyll. Sulfur ranked equal to nitrogen for optimizing crop yield and quality. It enhances the efficiency of nitrogen for protein manufacture (Tucker, 1999). The major reservoir of sulphur in soil is an unavailable elemental and reduced form of sulphur. Plants generally utilize the oxidised state of sulphate (SO4-2) and 
therefore, it must first be oxidized before it can be used by crops. Most agricultural soils contain some micro organisms that are able to oxidize sulphur. The micro organisms are mainly responsible to make available sulphate from elemental or reduced forms of sulphur through its oxidation process with the intermediate formation of thiosulphate (S2O32-) and tetrathionate (S4O62-) in soils (Anandham and Sridar, 2004). Sulphur oxidizing fungi appear particularly useful for oxidation purpose since their spores or mycelia can be produced economically in large quantities and have good survival characteristics, both in the inoculants and in soil. Reports showed that combined inoculation of Thiobacillus thioxidans and Aspergillus niger, Trichoderma harzianum, Myrothecium cinctum, Aspergillus terreus with sulphur improved soybean grain yield and protein content (Shinde et al., 2004). Therefore, the study was undertaken to determine performance of sulphur oxidizing fungi on yield parameters of soybean, available sulphur in soil and on sulphur uptake.

\section{Material and Method:}

The sulphur oxidizing fungi were isolated from soil samples collected from selected spots of different oilseed crops i.e. sesame, mustard, linseed and soybean fields of Agronomy Section, College of Agriculture, Nagpur. These isolates were further purified and screened for sulphur oxidizing ability in potato dextrose agar medium and czapek dox medium respectively. From these, two isolates i.e. Aspergillus niger and Trichoderma harzianum were selected for further experiment. A field experiment was conducted in randomized block design with three replications during kharif, 2012. There were nine treatments comprising of two fungal cultures and two sulphur levels (Table 1). The recommended dose of NPK (30:75:30 kg ha1) was applied in the form of DAP and MOP, sulphur in the form of elemental sulphur powder. The cultures were multiplied in modified sulphur medium (Wainwright, 1978) and when they attained 10-7 cfu ml-1 strength, each one was mixed in 1:2 (v/w) carrier. Then 
each of the cultures@250 g $10 \mathrm{~kg}-1$ of JS-335 seed of soybean were treated. The yield parameters of soybean viz., number of pods plant-1, grain yield and 1000 grains weight were studied. Five plants from each net plot were uprooted at harvest and all the developed pods were plucked and counted to estimate number of pods plant-1. The available sulphur content in soil and sulphur uptake by plant was analyzed by turbidimetric method (Chopra and Kanwar, 1980).

\section{Result and Discussion:}

The influence of sulphur oxiding fungal cultures with and without sulphur on the growth and yield of soybean plant was found to be significant. Particularly, inoculation of Trichoderma harzianum culture along with sulphur (15 kg ha-1) showed significantly superior performance over all other treatments under study. It is revealed from the data that number of pods plant- 1 were increased due to the cultures under study with and without sulphur over control. The maximum number of pods (68.34 plant-1) were recorded in the treatment Trichoderma harzianum culture and recommended dose of fertilizer along with sulphur (15 kg ha-1) and it was significantly superior over rest of the treatments and control. Next to this treatment application of Aspergillus niger culture and recommended dose of fertilizer along with sulphur (15 kg ha-1) significantly better than the remaining treatments except Trichoderma harzianum + recommended dose of fertilizer + Sulphur $(10 \mathrm{~kg}$ ha-1). The remaining less effective treatments were viz., Aspergillus niger + recommended dose of fertilizer + Sulphur (10 kg ha-1), Sulphur (20 kg ha-1) + recommended dose of fertilizer, Trichoderma harzianum + recommended dose of fertilizer, Aspergillus niger + recommended dose of fertilizer, recommended dose of fertilizer in a descending manner. The minimum number of pods (47.30 plant1) was recorded in uninoculated control. Similar trend of results was noticed in respect of grain yield. The maximum grain yield (1590 kg ha-1) was obtained in Trichoderma harzianum culture and recommended dose of fertilizer along with 
sulphur (15 kg ha-1) treatment and minimum weight of 1000 grains per treatment was also noticed to be the maximum (127.43 g) with the treatment of Trichoderma harzianum culture and recommended dose of fertilizer along with sulphur (15 kg ha-1). Shinde et al. (2004) in soybean reported that the number of pods, grain yield and 1000 grains weight were significantly increased due to combined inoculation of Thiobacillus thioxidans and Aspergillus niger, Trichoderma harzianum, Myrothecium cinctum, Aspergillus terreus with sulphur. These results were conformed during the present studies so far as $\mathrm{T}$. harzianum and A. niger are concerned. Shinde et al. (2000) in cotton reported that the number of picked bolls and seed yield were significantly increased due to combined inoculation of Scolecobasidium constrictum, Myrothecium cinctum, Aspergillus terreus, Thiobacillus thioparus and T. thiooxidans. The available sulphur content in soil increased due to inoculation of sulphur oxidizing fungi in conjunction with sulphur application. The maximum available sulphur (19.60 kg ha-1) was observed in the treatment of

Trichoderma harzianum culture and recommended dose of fertilizer along with sulphur (15 kg ha-1) and it was at par with Aspergillus niger culture and recommended dose of fertilizer along with sulphur (15 kg ha-1) treatment. Next to these treatments the treatments were applicaton of Trichoderma harzianum + recommended dose of fertilizer + Sulphur (10 kg ha-1) and Aspergillus niger + recommended dose of fertilizer + Sulphur (10 kg ha-1). The sulphur treatment alone also helped in increasing available sulphur level in soil from $13.36 \mathrm{~kg}$ ha1 to $17.40 \mathrm{~kg}$ ha-1. The less effective treatments were viz., Trichoderma harzianum + recommended dose of fertilizer and Aspergillus niger + recommended dose of fertilizer. The minimum available sulphur $(13.36 \mathrm{~kg}$ ha1) was recorded in uninoculated control. Kadam et al. (2004) and Shinde et al. (2010) in soybean found increased available sulphur content in soil due to combined inoculation of Thiobacillus thioxidans and Aspergillus niger, Trichoderma harzianum, Myrothecium cinctum, Aspergillus terreus with sulphur. The data on sulphur uptake by soybean crop revealed that two fungal 
cultures with or without sulphur treatment increased sulphur uptake ranging from 250.00 to $322.33 \mathrm{mg} 100-1 \mathrm{~g}$ dry weight of plant and maximum was recorded in Trichoderma harzianum culture and recommended dose of fertilizer along with sulphur (15 kg ha-1) and it was significantly superior over rest of the treatments. Next to this treatment the treatments were application of Aspergillus niger culture and recommended dose of fertilizer along with sulphur (15 kg ha-1), Trichoderma harzianum + recommended dose of fertilizer + Sulphur (10 kg ha-1) and Aspergillus niger + recommended dose of fertilizer + Sulphur (10 kg ha-1). The sulphur uptake also increased due to sulphur application alone from 250.00 to $262.00 \mathrm{mg} 100-1 \mathrm{~g}$ dry weight of plant. The less effective treatments were viz., Trichoderma harzianum + recommended dose of fertilizer and Aspergillus niger + recommended dose of fertilizer. The minimum sulphur uptake (250.00 mg 100-1 g) was recorded in uninoculated control. Shinde et al. (2004) reported increase in sulphur uptake in soybean due to combined inoculation of Thiobacillus thioxidans and Aspergillus niger, Trichoderma harzianum, Myrothecium cinctum, Aspergillus terreus with sulphur Earlier, Shinde et al. (2000) recorded similar results in groundnut and cotton crops. The significant effect of different cultures on the performance of soybean may be attributed to the increased rate of microbial oxidation of elemental sulphur to sulphate (SO4) sulphur in soil which in turn might have increased the sulphur uptake and thereby the yield.

\section{Conclusion:}

The influence of sulphur oxiding fungal cultures with and without sulphur on the growth and yield of soybean plant was found to be significant. Particularly, inoculation of Trichoderma harzianum culture along with sulphur (15 kg ha-1) showed significantly superior performance over all other treatments under study. 


\section{Acknowledgement:}

I wish to express my cordial thanks to respected scientist of the department of Plant Pathology for timely advice, coopretion, suggestion for this research work.

\section{References:}

Anandham, R. and R. Sridar, 2004. Use of sulfur bacteria for increased yield and oil content of groundnut. In: Biofertilizers Technology, Kannaiyan, S., K. Kumar and K. Govindarajan (Eds.). Jodhpur, Scientific Publishers, India. pp. 365-371.

Chopra, S. L. and J. S. Kanwar, 1980. Analytical Agricultural Chemistry. Kalyani Publ., New Delhi. pp. 259-260.

Kadam, R. M., D. B. Shinde, and A. C. Jadhav, 2004. Effects of sulphur oxidizing microorganisms on nodulation pattern of soybean. J. Maharashtra agric. Univ. 29 (3): 300-302.

Shinde, D. B., R. M. Kadam and A. C.Jadhav, 2004. Effects of sulphur oxidizing micro-organisms on growth of soybean. J. Maharashtra agric. Univ. 29 (3):305-307.

Shinde, D. B., A. C. Jadhav, R. M. Kadam, 2010. Effect of sulphur oxidizing microorganisms on nutrient availability in soil under soybean. J. Maharashtra agric. Univ. 35 (1): 113-116.

Tucker, M. R. 1999. Essential plant nutrients: Their presence in North Carolina soils and role in plant nutrition. Agronomic Division, NCDA and CS: pp. 20-25.

Wainwright, M. 1978. A modified sulphur medium for the isolation of sulphur oxidizing fungi. Plant and Soil. 49: 191-193. 
Table 1: Enumeration of Bacteria, Actinomycetes and Fugal population associated with different samples collected from various sites

\begin{tabular}{|c|c|c|c|c|c|c|c|}
\hline \multirow[b]{2}{*}{ LOCATION } & \multirow[b]{2}{*}{ SITES } & \multirow[b]{2}{*}{ SAMPLES } & \multicolumn{3}{|c|}{ Microbial count $\left(\times 10^{4} \mathrm{cfu} / \mathrm{g}\right.$ soil $)$} & \multirow{2}{*}{$\begin{array}{c}\text { Actinomycetes } \\
\text { count }\left(\times 10^{J} \text { cfu / }\right. \\
\text { g soil })\end{array}$} & \multirow[b]{2}{*}{$\begin{array}{c}\text { Fugal } \\
\text { count } \\
\left(\times 10^{5}\right. \\
\text { cfu / } \mathrm{g} \\
\text { soil })\end{array}$} \\
\hline & & & $\begin{array}{c}\text { Nutrient } \\
\text { Agar } \\
\text { (NA) }\end{array}$ & $\begin{array}{c}\text { Pikovaskaya's } \\
\text { agar (PVK) }\end{array}$ & $\begin{array}{c}\text { Soil } \\
\text { extract } \\
\text { media } \\
(\mathrm{SEM}) \\
\end{array}$ & & \\
\hline \multirow{15}{*}{ SOLAN } & \multirow{3}{*}{ NAUNI } & $\begin{array}{c}\text { Rural } \\
\text { compost }\end{array}$ & 121.00 & 83.00 & 106.13 & 67.11 & 12.13 \\
\hline & & $\begin{array}{l}\text { Mushroom } \\
\text { compost }\end{array}$ & 103.34 & 72.33 & 104.03 & 54.66 & 10.45 \\
\hline & & Soil & 126.34 & 84.33 & 116.01 & 55.12 & 17.11 \\
\hline & \multirow{3}{*}{ CHAIL } & $\begin{array}{l}\text { Rural } \\
\text { compost }\end{array}$ & 111.31 & 74.00 & 68.02 & 58.66 & 18.01 \\
\hline & & $\begin{array}{c}\text { Mushroom } \\
\text { compost }\end{array}$ & 90.64 & 53.67 & 62.00 & 33.15 & 13.21 \\
\hline & & Soil & 113.00 & 77.34 & 84.66 & 37.33 & 20.21 \\
\hline & \multirow{3}{*}{ CHAMBAGHAT } & $\begin{array}{l}\text { Rural } \\
\text { compost }\end{array}$ & 97.30 & 89.00 & 98.33 & 45.62 & 21.28 \\
\hline & & $\begin{array}{c}\text { Mushroom } \\
\text { compost }\end{array}$ & 76.33 & 54.00 & 72.03 & 28.12 & 15.11 \\
\hline & & Soil & 80.34 & 61.01 & 82.31 & 31.22 & 19.42 \\
\hline & \multirow{2}{*}{ SAPROON } & $\begin{array}{l}\text { Rural } \\
\text { compost }\end{array}$ & 78.03 & 59.10 & 100.13 & 51.00 & 22.31 \\
\hline & & Soil & 98.89 & 63.11 & 103.88 & 42.12 & 17.26 \\
\hline & BAROG & $\begin{array}{l}\text { Mushroom } \\
\text { compost }\end{array}$ & 121.76 & 78.33 & 111.65 & 34.27 & 11.34 \\
\hline & SALOGRA & $\begin{array}{l}\text { Municipal } \\
\text { solid waste }\end{array}$ & 87.12 & 34.12 & 45.13 & 21.21 & 8.34 \\
\hline & \multirow[t]{2}{*}{ DHARAMPUR } & $\begin{array}{l}\text { Rural } \\
\text { compost }\end{array}$ & 132.44 & 67.88 & 167.43 & 56.18 & 24.81 \\
\hline & & Soil & 104.54 & 44.32 & 93.24 & 39.23 & 15.23 \\
\hline \multirow{3}{*}{ SHIMLA } & \multirow[b]{2}{*}{ SHOGI } & $\begin{array}{l}\text { Rural } \\
\text { compost }\end{array}$ & 144.15 & 66.11 & 102.44 & 51.22 & 19.44 \\
\hline & & Soil & 98.66 & 76.65 & 113.77 & 44.31 & 17.63 \\
\hline & LAALPANI & $\begin{array}{l}\text { Municipal } \\
\text { solid waste }\end{array}$ & 77.22 & 34.62 & 55.53 & 19.34 & 9.45 \\
\hline
\end{tabular}


Table 2: Morphological and Biochemical characterization of the bacterial isolates

\begin{tabular}{|c|c|c|c|c|c|}
\hline \multirow[t]{2}{*}{ Isolates } & \multicolumn{3}{|c|}{ Morphology } & \multirow[t]{2}{*}{ Simple staining } & \multirow[t]{2}{*}{ Grams staining } \\
\hline & Form & Elevation & Margin & & \\
\hline DB1 & Irregular & Raised & Erose & Rods & + \\
\hline$\overline{\mathrm{DB} 2}$ & circular & Raised & Entire & Rods & + \\
\hline DB3 & Irregular & Flat & Undulate & Rods & + \\
\hline DB4 & Irregular & Flat & Erose & Rods & + \\
\hline DB5 & circular & Flat & Entire & Rods & + \\
\hline$\overline{\mathrm{DB} 6}$ & circular & Raised & Undulate & Rods & + \\
\hline DB7 & Irregular & Convex & Entire & Rods & + \\
\hline DB8 & circular & Convex & Entire & Rods & + \\
\hline DB9 & circular & Flat & Entire & Rods & + \\
\hline DB10 & circular & Flat & Undulate & Rods & + \\
\hline DB11 & Irregular & Raised & Undulate & Rods & - \\
\hline DB12 & circular & Convex & Entire & Rods & - \\
\hline DB13 & circular & Convex & Entire & Rods & - \\
\hline DB14 & Irregular & Flat & Erose & Rods & + \\
\hline DB15 & circular & Flat & Entire & Rods & + \\
\hline DB16 & Irregular & Raised & Entire & Rods & - \\
\hline DB17 & circular & Convex & Entire & Rods & + \\
\hline DB18 & circular & Flat & Undulate & Rods & + \\
\hline DB19 & circular & Flat & Undulate & Rods & - \\
\hline DB20 & Irregular & Flat & Erose & Rods & + \\
\hline DB21 & circular & Convex & Entire & Rods & + \\
\hline DB22 & circular & Raised & Undulate & Rods & - \\
\hline DB23 & circular & Convex & Entire & Rods & + \\
\hline DB24 & Irregular & Raised & Erose & Rods & + \\
\hline DB25 & Irregular & Flat & Undulate & Rods & + \\
\hline DB26 & Irregular & Flat & Undulate & Rods & + \\
\hline
\end{tabular}


Table 3: Biochemical characteristics of the bacterial isolates

\begin{tabular}{|c|c|c|c|c|c|c|c|c|}
\hline Isolates & $\begin{array}{c}\text { Catalase } \\
\text { test }\end{array}$ & $\begin{array}{c}\text { Oxidase } \\
\text { test }\end{array}$ & $\begin{array}{l}\text { MR } \\
\text { test }\end{array}$ & $\begin{array}{l}\text { VP } \\
\text { test }\end{array}$ & $\begin{array}{l}\text { Carbohydrate } \\
\text { fermentation }\end{array}$ & $\begin{array}{c}\text { Casein } \\
\text { hydrolysis }\end{array}$ & $\begin{array}{l}\text { Gelatin } \\
\text { hydrolysis }\end{array}$ & $\begin{array}{c}\text { Hydrogen } \\
\text { sulphide } \\
\text { production }\end{array}$ \\
\hline DB1 & + & - & - & + & + & + & + & - \\
\hline DB2 & + & - & - & + & + & - & + & - \\
\hline DB3 & + & - & - & + & + & - & + & - \\
\hline DB4 & + & - & - & + & + & - & + & - \\
\hline DB5 & + & - & - & + & + & + & + & - \\
\hline DB6 & + & - & - & + & + & + & - & - \\
\hline DB7 & + & - & - & + & + & + & + & - \\
\hline DB8 & + & - & - & + & + & + & + & - \\
\hline DB9 & + & - & - & + & + & - & + & - \\
\hline DB 10 & + & - & - & + & + & - & + & - \\
\hline DB11 & - & - & - & + & + & - & - & - \\
\hline DB12 & - & - & - & + & + & + & + & - \\
\hline DB13 & + & - & - & + & + & + & + & - \\
\hline DB14 & + & - & - & + & + & + & - & - \\
\hline DB15 & + & - & - & + & + & + & + & - \\
\hline DB16 & + & - & - & + & + & - & + & - \\
\hline DB17 & + & - & - & + & - & + & - & - \\
\hline DB18 & + & - & - & + & + & + & + & - \\
\hline DB19 & + & - & - & + & + & + & + & - \\
\hline DB20 & + & - & - & + & + & - & + & - \\
\hline DB21 & + & - & - & + & + & + & + & - \\
\hline DB22 & + & - & - & + & - & - & - & - \\
\hline DB23 & + & - & - & + & + & + & - & - \\
\hline DB24 & + & - & - & + & + & + & + & - \\
\hline DB25 & + & - & - & + & + & - & + & - \\
\hline DB26 & + & - & - & + & + & - & + & - \\
\hline
\end{tabular}


Table 4: Screening of selected bacterial isolates for multifarious plant growth promoting traits

\begin{tabular}{|c|c|c|c|c|c|c|c|}
\hline \multirow[b]{2}{*}{ Isolates } & \multirow{2}{*}{$\underset{\text { Solubilization }}{\mathbf{P}}$} & \multirow{2}{*}{$\begin{array}{c}\text { Growth } \\
\text { on } \mathbf{N} \\
\text { free } \\
\text { medium }\end{array}$} & \multirow{2}{*}{$\begin{array}{c}\mathrm{HCN} \\
\text { production }\end{array}$} & \multirow{2}{*}{$\begin{array}{l}\text { Siderophore } \\
\text { production }\end{array}$} & \multirow[b]{2}{*}{ Cellulolytic } & \multicolumn{2}{|c|}{ Antagonism against } \\
\hline & & & & & & $\begin{array}{l}\text { Fusarium } \\
\text { oxysporum }\end{array}$ & $\begin{array}{l}\text { Pythium } \\
\text { ultimum }\end{array}$ \\
\hline DB1 & +++ & ++ & +++ & ++ & + & + & + \\
\hline DB2 & ++ & ++ & - & ++ & - & - & - \\
\hline DB3 & + & + & + & - & + & + & - \\
\hline DB4 & ++ & - & +++ & + & - & - & - \\
\hline DB5 & ++ & +++ & - & + & + & + & - \\
\hline DB6 & - & - & ++ & +++ & - & + & - \\
\hline DB7 & +++ & + & + & ++ & - & - & - \\
\hline DB8 & - & ++ & ++ & - & - & - & - \\
\hline DB9 & - & - & + & + & + & + & - \\
\hline DB10 & ++ & + & - & +++ & - & + & - \\
\hline DB11 & ++ & + & + & - & + & - & - \\
\hline DB12 & - & ++ & ++ & + & - & + & - \\
\hline DB13 & + & + & - & + & - & + & - \\
\hline DB14 & +++ & +++ & + & +++ & - & - & - \\
\hline DB15 & - & - & - & ++ & - & + & - \\
\hline DB16 & - & +++ & - & ++ & + & - & + \\
\hline DB17 & ++ & - & - & + & - & - & - \\
\hline DB18 & ++ & +++ & + & - & - & - & + \\
\hline DB19 & - & - & ++ & - & - & + & - \\
\hline DB20 & +++ & - & - & ++ & + & - & + \\
\hline DB21 & - & - & +++ & +++ & - & + & - \\
\hline DB22 & ++ & +++ & + & + & - & - & + \\
\hline DB23 & ++ & - & - & - & - & - & + \\
\hline DB24 & + & ++ & - & - & - & - & + \\
\hline DB25 & +++ & ++ & - & ++ & - & - & - \\
\hline DB26 & + & ++ & ++ & +++ & - & + & - \\
\hline
\end{tabular}

${ }^{*}$ values ranging from 55-75 \% (++), $\leq 55 \%(+), \geq 75 \%(+++)$, no activity (-) ; ** values ranging from $20-25(++), \leq 20(+), \geq 25(+++)$, no activity $(-) ; * * *$ values ranging from 50-80 \% $(++), \leq 50 \%(+), \geq 80 \%(+++),(-)$ no activity ; \# Values ranging from $20-35(++), \leq 20(+), \geq 35(+++)$, no activity $(-)$ 
Table 5: Screening of selected actinomycelial isolates for multifarious plant growth promoting traits

\begin{tabular}{|c|c|c|c|c|c|}
\hline Isolates & P solubilization & $\begin{array}{l}\text { Growth on N } \\
\text { free medium }\end{array}$ & $\begin{array}{c}\text { HCN } \\
\text { production }\end{array}$ & $\begin{array}{c}\text { Siderophore } \\
\text { production }\end{array}$ & Cellulolytic \\
\hline$\overline{\text { DA1 }}$ & +++ & - & - & ++ & + \\
\hline DA 2 & ++ & +++ & - & + & - \\
\hline DA 3 & +++ & - & - & - & - \\
\hline$\overline{\mathrm{DA}} 4$ & ++ & + & ++ & + & - \\
\hline DA 5 & ++ & +++ & + & - & - \\
\hline DA 6 & + & - & ++ & +++ & - \\
\hline DA 7 & +++ & ++ & ++ & +++ & + \\
\hline DA 8 & - & ++ & ++ & - & - \\
\hline DA 9 & +++ & - & + & + & - \\
\hline DA 10 & ++ & + & - & +++ & - \\
\hline DA 11 & +++ & ++ & - & - & - \\
\hline DA 12 & - & - & +++ & +++ & - \\
\hline DA 13 & +++ & ++ & - & +++ & - \\
\hline DA 14 & - & - & + & ++ & - \\
\hline DA 15 & - & - & - & ++ & + \\
\hline DA 16 & - & ++ & - & ++ & - \\
\hline DA 17 & +++ & ++ & +++ & ++ & - \\
\hline
\end{tabular}

Table 6: Screening of selected fungal isolates for multifarious plant growth promoting traits

\begin{tabular}{|c|c|c|c|c|c|}
\hline Isolates & P solubilisation & $\begin{array}{l}\text { Growth on N } \\
\text { free medium }\end{array}$ & HCN production & $\begin{array}{c}\text { Siderophore } \\
\text { production }\end{array}$ & Cellulolytic \\
\hline$\overline{\mathrm{DF} 1}$ & ++ & ++ & + & ++ & - \\
\hline DF2 & +++ & + & - & ++ & - \\
\hline DF3 & + & + & + & - & + \\
\hline DF4 & +++ & - & +++ & + & - \\
\hline DF5 & ++ & +++ & + & - & - \\
\hline DF6 & + & - & ++ & +++ & - \\
\hline DF7 & ++ & - & + & ++ & + \\
\hline DF8 & - & ++ & ++ & - & - \\
\hline DF9 & +++ & - & + & + & + \\
\hline$\overline{\text { DF10 }}$ & ++ & + & - & +++ & - \\
\hline DF11 & +++ & ++ & - & - & - \\
\hline DF12 & ++ & ++ & + & ++ & + \\
\hline DF13 & - & ++ & ++ & + & + \\
\hline DF14 & +++ & ++ & ++ & +++ & + \\
\hline DF15 & ++ & - & - & ++ & - \\
\hline DF16 & - & ++ & - & ++ & + \\
\hline DF17 & +++ & ++ & - & ++ & - \\
\hline DF18 & ++ & ++ & + & + & - \\
\hline DF19 & - & - & ++ & - & - \\
\hline DF20 & +++ & - & - & ++ & + \\
\hline DF21 & - & - & +++ & +++ & - \\
\hline DF22 & ++ & +++ & - & + & - \\
\hline DF23 & +++ & - & - & - & - \\
\hline
\end{tabular}


Table 7: Dual culture compatibility assessment amongst eleven bacterial isolates of tomato seedlings

\begin{tabular}{|c|c|c|c|}
\hline $\begin{array}{c}\text { Bacterial } \\
\text { Isolates }\end{array}$ & $\begin{array}{c}\text { Synergism with } \\
\text { actinomycetes isolates }\end{array}$ & $\begin{array}{c}\text { Synergism with fungal } \\
\text { isolates }\end{array}$ & Compatibility \\
\hline DB1 & DA7 & DF14 & + \\
\hline DB1 & DA17 & DF12 & - \\
\hline DB1 & DA7 & DF12 & - \\
\hline DB1 & DA17 & DF14 & - \\
\hline DB 22 & DA7 & DF14 & - \\
\hline DB 22 & DA17 & DF12 & - \\
\hline DB 22 & DA7 & DF12 & + \\
\hline DB 22 & DA17 & DF14 & \\
\hline
\end{tabular}

Table 7: Dual culture compatibility assessment amongst eleven bacterial isolates of tomato seedlings 